\title{
Retraction notice for: "MicroRNA-221 promotes cell proliferation, migration, and differentiation by regulation of ZFPM2 in osteoblasts" [Braz J Med Biol Res (2018) 51(12): e7574]
}

\author{
Xingguo Zheng ${ }^{1}$, Jinhua Dai $^{2}$, Haijun Zhang ${ }^{1}$, and Zhibin $\mathrm{Ge}^{1}$ \\ ${ }^{1}$ Department of Orthopaedics, Ningbo No. 2 Hospital, Ningbo, China \\ ${ }^{2}$ Department of Clinical Laboratory, Ningbo No. 2 Hospital, Ningbo, China
}

Retraction for: Braz J Med Biol Res | doi: 10.1590/1414-431X20187574 | PMCID: PMC6207289 | PMID: 30365725

On April 15, 2020, the Brazilian Journal of Medical and Biological Research (BJMBR) received a request from the Corresponding author Zhibin Ge to withdraw this manuscript: "In a subsequent experiment, we repeated the experiment in this manuscript. Unfortunately, we had different results than before. This led to inaccurate conclusions in the manuscript. In order to ensure the accuracy of the articles we have published, we must request retraction. All authors agree to this retraction".

As per consensus between the Editors-in-Chief of the BJMBR and the Authors, the article has been retracted. 


\title{
MicroRNA-221 promotes cell proliferation, migration, and differentiation by regulation of ZFPM2 in osteob asts
}

\author{
Xingguo Zheng ${ }^{1}$, Jinhua Dai ${ }^{2}$, Haijun Zhang ${ }^{1}$ a ' Zhibin Ge' \\ ${ }^{1}$ Department of Orthopaedics, Ningbo No. 2 , Chinal, Nin \\ ${ }^{2}$ Department of Clinical Laboratory, Ningbo No. Hospital Ningoo, China
}

\begin{abstract}
Bone fracture is a common medical condition, which may occur due to traumatic injury dist -related conditions. Evidence suggests that microRNAs (miRNAs) can regulate osteoblast differentiation and function this dy, we explored the effects and mechanism of miR-221 on the growth and migration of osteoblasts using MC3 ${ }^{\top}<1$ lls. The expression levels of miR-221 in the different groups were measured by qRT-PCR. Then, miR-221 mimic and bitc ansfected into MC3T3-E1 cells, and cell viability and migration were measured using the CCK-8 assay and the swell migration assay. Additionally, the expression levels of differentiation-related factors (Runx2 and Ocn) and $7-D M 2$ wer easured by qRT-PCR. Western blot was used to measure the expression of cell cycle-related proteins, epithe $1-1$. ZFPM2, and Wnt/Notch, and Smad signaling pathway proteins. miR-221 s gnimcantly up-regulated in the patients with lumbar compression fracture (LCM) and trochanteric fracture (TF). miR-221 moted ALP, Runx2, and OPN expressions in MC3T3-E1 cells. miR-221 overexpression significantly increased onll proliferatic , migration, differentiation, and matrix mineralization, whereas suppression of miR-221 reversed these effe. An inally, the results displayed that ZFPM2 was a direct target gene of miR-221, and overexpression of ZFPM2 rev $2 d$ the $p$ moting effects of miR-221 overexpression on osteoblasts. Mechanistic study revealed that overexpression of iR- $\llcorner$ inac ated the Wnt/Notch and Smad signaling pathways by regulating ZFPM2 expression. We drew the conclusions at miR-2 2 rexpression promoted osteoblast proliferation, migration, and differentiation by regulation of ZFPM2 expressic and eactivating the Wnt/Notch and Smad signaling pathways.
\end{abstract}

Key words: MicroRNA-221; Bone fracture; Oste slast dit ation; ZFPM2; Wnt/Notch; Smad

\section{Introduction}

Bone fracture is a common and creasi disease, which results from both traumatic injury dis se-related bone fragility (1). In the Unite States, $x$ six million adults suffer from fractures o van 20 , Bone fracture may lead to fever, disability, sh $k$ nd treatment is very expensive. Timely and pria management of bone fractures can help pa' nts store iginal functions. However, some patients $s$. such as osteom itis, $\mathrm{h}$ union and mal-union, complex regional pain y 'rome, and post-traumatic arthritis (3). Bone fracture nealin a physiologically complex process, which in ves both blu ogical and mechanical factors (4). Follow bc fracture, a series of events occurs, including cell 1 ation ifferentiation, tissue synthesis, and the $r$ of anes and growth factors. The recovery oce of fracture depends on the activity of osteoblasts uscublasts are mesenchymal cells, which play a maj role in skeletal development and bone formation (7). Osteoulasts are responsible for the synthesis, secretion and mineralization of bone matrix (8). Therefore, it is necessary to explore the mechanism of osteoblast proliferation, migration, and differentiation.

MicroRNAs (miRNAs) are small, non-coding RNA molecules, which can mediate the post-transcriptional gene expression (9). A recent study demonstrates that miRNAs are involved in various cellular processes, such as cell proliferation, migration, differentiation, and apoptosis (10). Increasing evidence indicates that miRNAs regulate the differentiation and function of chondrocytes, osteoblasts, and osteoclasts (11). These findings suggest that miRNAs act as key mediators in the processes of bone formation, resorption, remodeling, and repair (12). As Waki et al. (13) reported, several miRNAs, such as miR-140-3p, miR-140-5p, miR-181a-5p, miR-181d-5p, and miR-451a, were significantly up-regulated in standard healing fractures compared with unhealing fractures. Moreover, more than 15 miRNAs have been reported for bone formation stimulation (14). miR-221 is one of many widely studied miRNAs, which is frequently up-regulated in various cancers $(15,16)$. However, the precise function of miR-221 in bone fracture is

Correspondence: Zhibin Ge: <gezhibin315@126.com>

Received April 19, 2018 | Accepted August 13, 2018 
still unknown. In this study, we aimed to explore the role of miR-221 in osteoblast proliferation, migration, and differentiation using MC3T3-E1 cells. This is the first study to report the effects of miR-221 on osteoblast growth and differentiation in the bone fracture healing process. This study might provide novel therapeutic strategies for bone fracture.

\section{Material and Methods}

\section{Blood sample collection}

The blood samples were obtained from three patients with lumbar compression fracture (LCM) and three patients with trochanteric fracture (TF) of Ningbo No.2 Hospital from April 2017 to October 2017. The blood samples were collected at $24 \mathrm{~h}$ and 7,14 , and 21 days after surgery or injury and then stored at $-80^{\circ} \mathrm{C}$ until analyzed. The ethical approval for this study was granted by the Ethics Committee of Ningbo No. 2 Hospital. All participants signed the informed consent.

\section{Cell culture and differentiation induction}

The mouse osteoblast-like cells (MC3T3-E1) used in the present study were obtained from American Type Culture Collection (ATCC, USA). The cells were cultured in $100-\mathrm{mm}$ dishes containing $\alpha$-MEM culture me am with $10 \%$ fetal bovine serum (FBS, Gibco, USA), d $1^{\circ}$ penicillin and streptomycin under humid environ $\eta$

$5 \% \mathrm{CO}_{2}$ at $37^{\circ} \mathrm{C}$. The culture medium was ch ged en three days. For induction of MC3T3-E1 cells $1 \times 10^{5}$ cells were seeded in a 6 -well plate, and in hated in the differentiation medium containin $\mu \mathrm{g} / \mathrm{mL}$ ascurbic acid and $10 \mathrm{mmol} / \mathrm{L} \beta$-glycerophosphe The $\mathrm{c}$ trol group cells were cultured with conventional $n$ dium. 7 e medium was refreshed every three day

\section{Quantitative real-time polyme sharn reaction (qRT-PCR) analysis}

Total RNA was is ated om b. od samples using the PAXgene Blood RN. MC3T3-E1 cells sing common kit of Trizol reagent (Invitrogen, Lis chnolog. Corporation, USA) according to manufacture rotocols. For examining the expression leve of miR-2 in MC3T3-E1 cells, cDNA was synthe ed ing TaqMan MicroRNA Reverse Transcription Kit uroge , and qRT-PCR analysis was carried o ing an Universal Master Mix II (Invitrogen) slow a the nstructions, and U6 (Applied Biosystems, wru ased to normalized the expression level of mi 21. Data were examined by the $2^{-\Delta \Delta \mathrm{Ct}}$ method.

\section{miRNAs transfection}

The expression plasmids of miR-221 mimic, miR-221 inhibitor, and the corresponding negative controls (NCs) were synthesized by GenePharma Co. (China). Additionally, the overexpression vector of zinc finger protein multitype 2
(ZFPM2) was constructed using the sub-cloning the fulllength ZFPM2 coding sequence into pcDNA3.1 asmid (Sangon Biotech, China). The empty pcDNA3 pla nid was used as a negative control. Afterward, 'C' $3-E$ ' cells were transfected with these expression pla is $48 \mathrm{~h}$. All cell transfections were detected using Lipc. tamine 3000 reagent (Invitrogen) accora to the nanufacturer's protocol.

\section{Cell viability assay}

Cell viability of MC3T3-E Cell Counting Kit-8 (CCK) as (Beyotime Biotechnology, China). Briefly, N T3-E1 a were cultured in a 96-well plate, and traisfe with expression vectors of miR-221 mimic, $r^{-} 221$ inh $^{-}$or, and pc-ZFPM2. After transfection for $\sigma \mathrm{h}, 0 \mu \mathrm{L} \mathrm{CCK}-8$ was supplemented to each well anc ere incubated for another $1 \mathrm{~h}$ under the routine ture environment containing 95\% air and $5 \%+37^{\circ} \mathrm{C}$. inally, a microplate reader (Bio-Rad, USA) wa. Ise easure the absorbance at $450 \mathrm{~nm}$.

\section{Cell migrat assay}

or the migration assay, the Transwell with a pore size of 8 , 1 was performed to examine the migration ability of MC 「3-E1 cells. In brief, MC3T3-E1 cells were transto with the above expression vectors. Afterward, these celı were suspended in serum-free medium, and $100-\mu \mathrm{L}$ ell suspension was added into the upper compartment of a 24-well transwell culture chamber. Meanwhile, $600 \mu \mathrm{L}$ of complete medium was added into the lower compartment. After incubation for $24 \mathrm{~h}$ in the conventional culture conditions, the Transwell culture chamber was taken out, and washed twice with calcium-free PBS, and cells were fixed with methanol for $30 \mathrm{~min}$. Subsequently, the non-migrated cells were removed carefully using a wet cotton swab from the upper surface of the filter. The migrated cells on the lower side of the filter were stained with $1 \%$ crystal violet for $20 \mathrm{~min}$, and counted using a microscope (magnification of $400 \times$ ) in a random five fields of vision.

\section{Alizarin Red S staining assay}

To confirm the important effects of miR-221 on mineralization of MC3T3-E1 cells, the Alizarin Red S staining assay was performed. Briefly, the cells were washed twice with PBS and fixed with $95 \%$ ethanol for $10 \mathrm{~min}$ at room temperature. Subsequently, the fixed cells were stained with $1 \%$ Alizarin Red S solution (Sigma-Aldrich, USA) for $30 \mathrm{~min}$ at $37^{\circ} \mathrm{C}$ and counted using a light microscope (Olympus, Japan). Quantification of Alizarin Red $\mathrm{S}$ stain was assessed via extraction with Image $\mathrm{J}$ software (NIH, USA).

\section{Luciferase reporter assay}

The $3^{\prime}$-untranslated region $\left(3^{\prime}\right.$-UTR) of ZFPM2 was amplified by PCR and inserted into pmiR-Report vector (Promega, USA). Then, the vectors were co-transfected 
with miR-221 mimic and its corresponding control into cells using Lipofectamine 3000 (Invitrogen). The luciferase assay was confirmed using the dual luciferase reporter assay system (Promega) after transfection for $48 \mathrm{~h}$.

\section{Western blot assay}

The proteins from transfected cells used for the western blot assay were isolated using RIPA lysis buffer (Beyotime Biotechnology). The contents of total protein were tested using the $\mathrm{BCA}^{\mathrm{TM}}$ Protein Assay Kit (Pierce, USA) based on the kit instruction. Then, $40 \mu \mathrm{g}$ protein samples were subjected to sodium dodecyl sulfate-polyacrylamide gel electrophoresis (SDS-PAGE) and transferred to polyvinylidene difluoride (PVDF) membranes. After blocking with $5 \%$ non-fat milk, the membranes were transferred to another container and incubated with the primary antibodies of alkaline phosphatase (ALP, ab83259), Runt-related transcription factor 2 (Runx2, ab23981), Osteopontin (OPN, ab8448), E-cadherin (ab40772), N-cadherin (ab18203), Vimentin (ab16700), ZEB1 (ab124512), Snail (ab82846), Osteocalcin (Ocn, ab93876), proliferating cell nuclear antigen (PCNA, ab18197), Cyclin A (ab181591), Cyclin E1 (ab71535), cyclin-dependent kinase 2 (CDK2, ab64669), Cyclin D1 (ab134175), CDK4 (ab199728), Wnt3a (ab28472) Wnt5a (ab229200), Notch 1 (ab52627), Notch 2 (ab8926), Notch 3 (ab23426), p-Smad2 (ab53100), Smad2 (ab33 5), Smad4 (ab40759), Smad7 (ab216428), and $C$.PD' (ab181602) at $4^{\circ} \mathrm{C}$ overnight. After incubation or at $37^{\circ} \mathrm{C}$, the secondary antibody of horseradish peroxidase (HRP)-conjugated goat anti-rabbit IgG (ab205718 .2000, Abcam) was added and incubated for $1 \mathrm{~h}$ at roor em rature. Finally, the signals were captured using $E$ re gents (MultiSciences Biotech, China).

\section{Statistical analysis}

The results are reported as $\mathrm{m} \pm \mathrm{S}_{\mathrm{C}} \mathrm{S} 19.0$ statistical software (SPSS, Inc., U A) was usea io analyze the data. One-way analysis of ariance ANOVA) was used to calculate the $P$ value onsidered to be statistically significant

\section{Results}

miR-221 was rec lated during osteoblast

\section{differentiatio}

To explore the fect of miR-221 on bone fracture, the blood $\mathrm{c}$ atration $\mathrm{f}$ miR-221 in three LCM patients and three $\mathrm{FP}$ were examined. The results showed that the bl concentrations of miR-221 were obviously increased $a \quad 7$ and 14 days after surgery, whereas the entrations of miR-221 was recovered at 21 days after surg, (Figure $1 \mathrm{~A}$ and $\mathrm{B}$ ). Additionally, MC3T3-E1 cells were Itured in osteogenic differentiation medium, and ative expression of miR-221 during the osteoblast diturentiation process was detected at different time interals $(0,4,7,14,21$, and 28 days) using qRT-PCR.
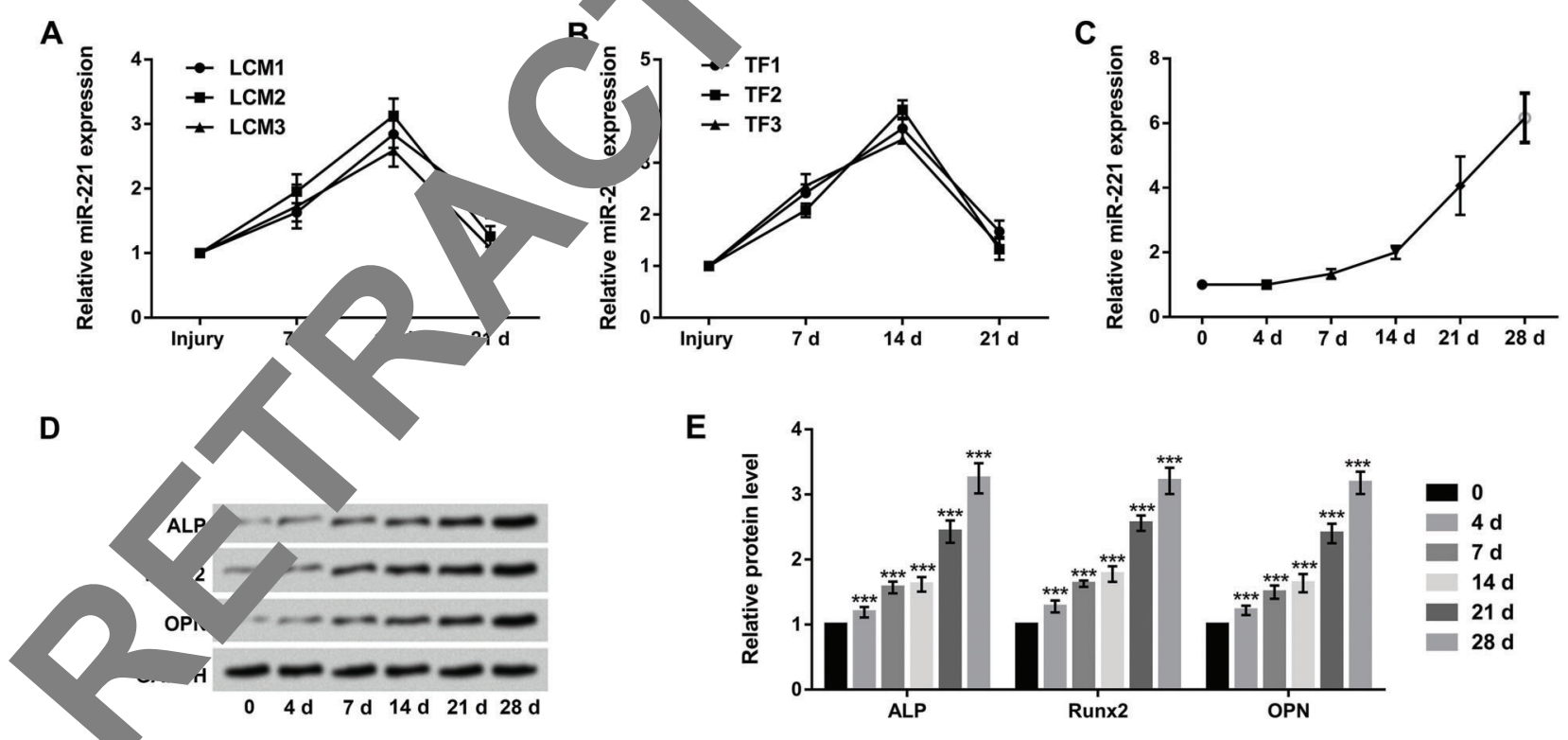

Figure 1. miR-221 is up-regulated during the osteoblast differentiation process. $A$ and $B$, Blood concentrations of miR-221 in three lumbar compression fracture (LCM) patients and three trochanteric fracture (TF) patients were examined by qRT-PCR. Then, MC3T3-E1 cells were treated with osteogenic differentiation media. C, Expression of miR-221 during osteoblast differentiation at $0,4,7,14,21$, and 28 days was detected by qRT-PCR. $D$ and $E$, Protein levels of ALP, Runx2, and OPN were assessed by western blot. miR-221: microRNA-221; qRT-PCR: quantitative reverse transcription polymerase chain reaction; ALP: alkaline phosphatase; Runx2: runt-related transcription factor 2; OPN: osteopontin. Data are reported as means $\pm S D$. ${ }^{\star \star *} P<0.001$ compared to baseline (ANOVA). 
A

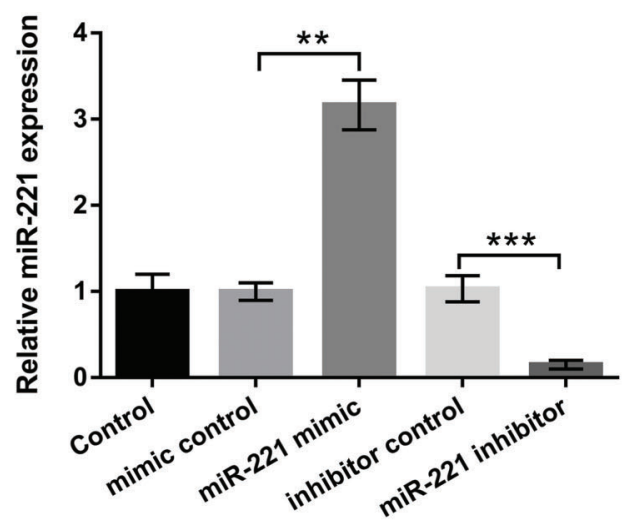

C

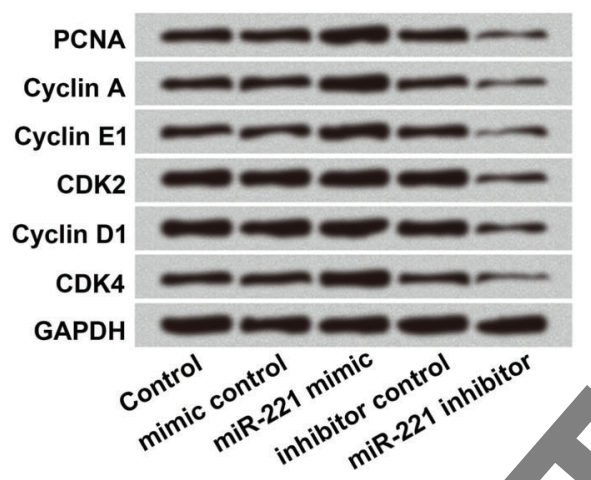

B

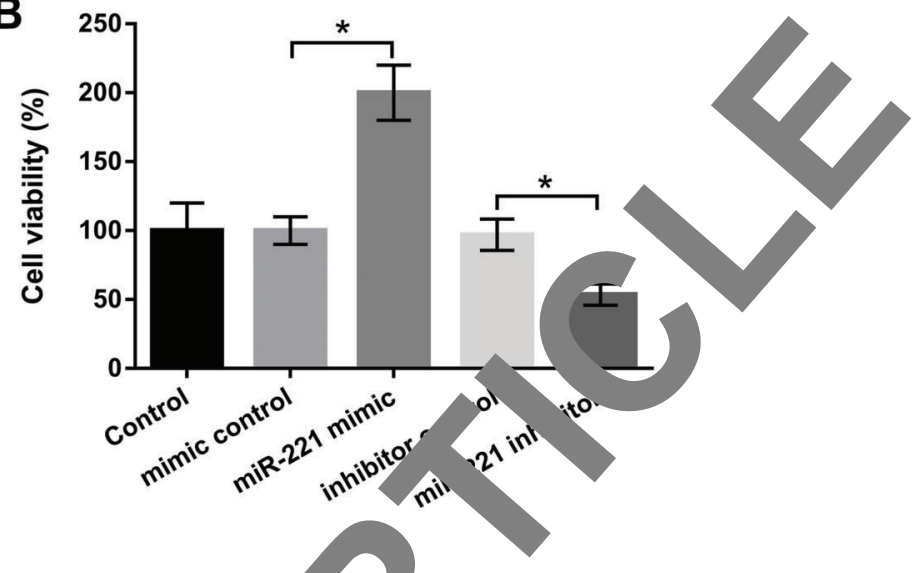

Figure 2. Overexpression of miR-221 prom osteoblas proliferation. $A$, Expression of miR-221 in transfection of MC3T3-E1 cells was detected using qRT-PCR. $B$, Cell vial yy was measured using the CCK-8 assay. $C$ and $D$, Western blot was used to measure the expression of cell cycle-related proteins. $m$-221: mI RNA-221; qRT-PCR: quantitative reverse transcription polymerase chain reaction; CCK-8: cell counting kit-8; PCNA: prolifera cell $r$ clear antigen; CDK: cyclin-dependent kinase; qRT-PCR: quantitative reverse tran-

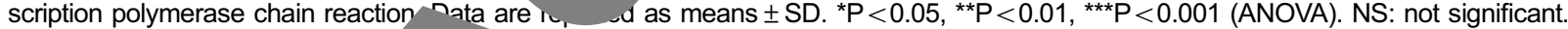

As shown in Figure 1C, the ex er or of miR-221 was significantly increased ir me-c endent manner during osteoblastic differenti on. arther, he western blot assay revealed that the pro in Runx2, and OPN were notably ur egula in a time-dependent manner $(P<0.001$, Fir 1D ana $)$. These data indicated that up-regulation of $\mathrm{m}_{\mathrm{h}} \quad 21 \mathrm{might}$ have an important role in the proce, of bone th ture.

Overex sion miR-221 promoted osteoblast pr ratic

Nf t. MCo 3-E1 cells were transfected with miR-221 $\angle 21$ inhibitor, and the corresponding controls. At. ransfection for $48 \mathrm{~h}$, the expression level of miR-221 was nusured using qRT-PCR. The results in Figure 2A showed that miR-221 overexpression significantly increased the expression of miR-221 compared with the mimic control group $(P<0.01)$, while miR-221 suppression significantly decreased the expression of miR-221 compared with the inhibitor control group $(P<0.001)$. We then measured cell viability using the CCK-8 assay. As shown in Figure 2B, miR-221 overexpression significantly promoted cell viability compared with the mimic control group $(P<0.05)$, whereas miR-221 suppression significantly decreased cell viability compared with the inhibitor control group $(P<0.05)$. Western blot assay was performed to further confirm these results by analysis of cell cyclerelated proteins (PCNA, Cyclin A, Cyclin E1, CDK2, Cyclin D1, and CDK4). The results showed that miR-221 overexpression increased the expression of these proteins, whereas miR-221 inhibition decreased their expression in MC3T3-E1 cells $(P<0.01$, Figure $2 C$ and $D)$. Taken together, the data indicated that overexpression of miR-221 could promote cell proliferation.

\section{Overexpression of miR-221 promoted osteoblast migration}

The results of the Transwell migration assay showed that miR-221 overexpression remarkably increased cell migration compared with the mimic control group $(P<0.05)$, 
A

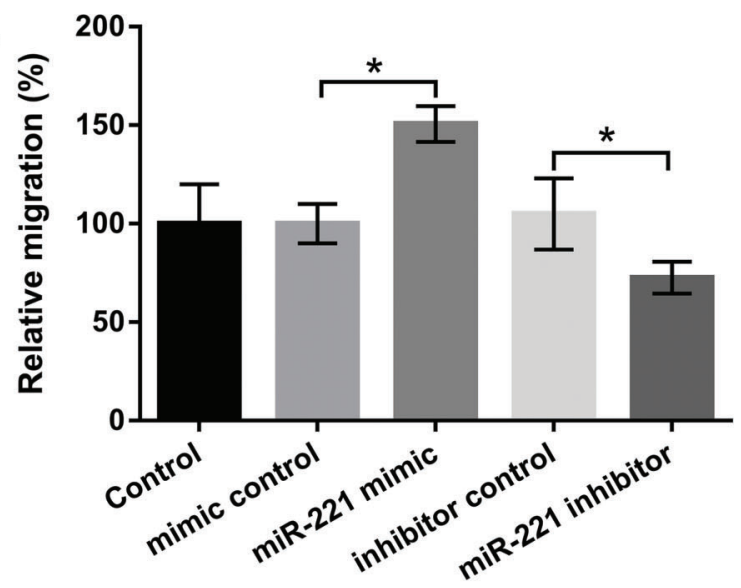

B

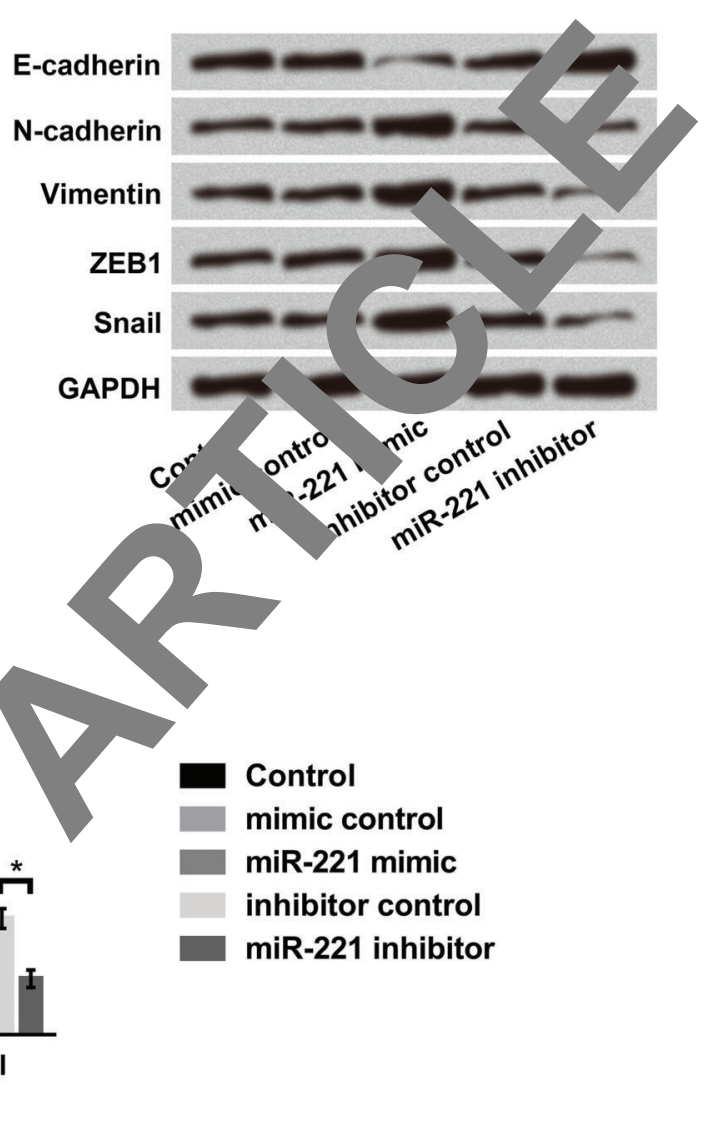

Figure 3. Overexpression of miR-221 promotes osteob migration. $A$, Cell migration was measured using the Transwell migration assay. $B$ and $C$, Protein levels of EMT-relate cueins (E-caanerin, $\mathrm{N}$-cadherin, Vimentin, ZEB1, and Snail) were determined using western blot. miR-221: microRNA-221; EMT: epithe 1-mesen ${ }^{*} y m a l$ transition. Data are reported as means $\pm S D$. ${ }^{*} P<0.05,{ }^{* *} P<0.01$ (ANOVA).

whereas miR-221 suppression significa. migration compared with ti hitor control group $(P<0.05$, Figure 3A). Western If esuits showed that miR-221 overexpressio ecre d the expression of E-cadherin $(P<0.01)$ as ll as creased the expressions of $\mathrm{N}$-cadherin, ne $\quad \mathrm{s}$, and Snail $(\mathrm{P}<0.01)$. However, miR-2 sup ssion showed opposite results (Figure 3B ar $\checkmark$. Thes indings indicated that overexpression or mik 1 could promote cell migration and EMT pror os in ostec asts.

Overex sion miR-221 promoted osteoblast dif ntia.

$\mathrm{qF}$-PCR esults of cell differentiation showed that $R$ Crexpression significantly increased the expro ion of Runx2 and Ocn on days 7 and 14, whereas miR- 1 suppression showed opposite results $(P<0.05$, Figure $4 \mathrm{~A}$ and $\mathrm{B}$ ). Mineralization analysis showed that overexpression of miR-221 significantly increased the mineralized nodule formation compared with the control group $(P<0.01$, Figure $4 C)$. Furthermore, the results of western blot (Figure 4D-F) revealed that miR-221 overexpression notably increased the protein levels of ALP, Runx2, and Ocn on days 7 and 14, whereas miR-221 suppression showed opposite results except for Ocn expression on day 14, which was unchanged. Above all, the results suggested that overexpression of miR-221 was associated with osteoblast differentiation.

\section{ZFPM2 was a direct target of miR-221}

To explore the relationship between miR-221 and ZFPM2, the software programs of TargetScan (www.target scan.org) and microRNA database (www.microrna.org) were used to predict the binding site (Figure 5A). Then, the expression level of ZFPM2 in MC3T3-E1 cells transfected with miR-221 mimic and miR-221 inhibitor was detected by qRT-PCR and western blot. The results showed that the mRNA and protein levels of ZFPM2 were significantly decreased by miR-221 overexpression, as well as promoted by miR-221 suppression ( $P<0.05$, Figure $5 B$ ). Meanwhile, dual-luciferase reporter assay results showed that luciferase activity was greatly decreased by co-transfection of miR-221 mimic and ZFPM2-WT $(P<0.05)$. However, cotransfection of miR-221 mimic and ZFPM2-MUT had no 
A

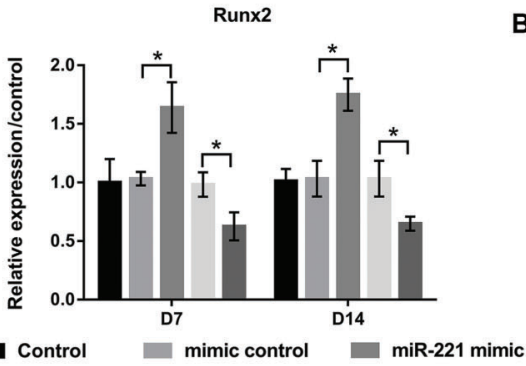

B

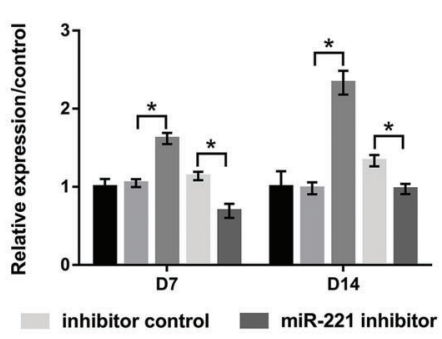

C

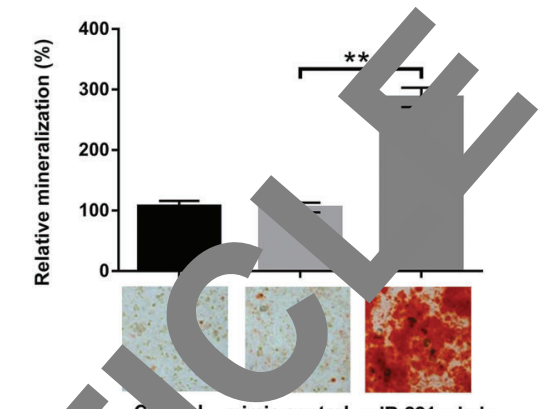

D

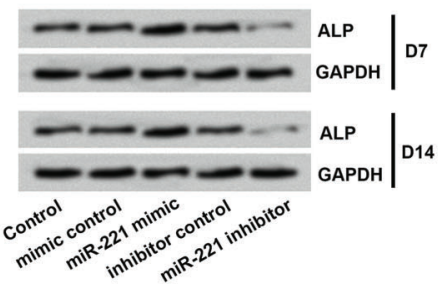

E

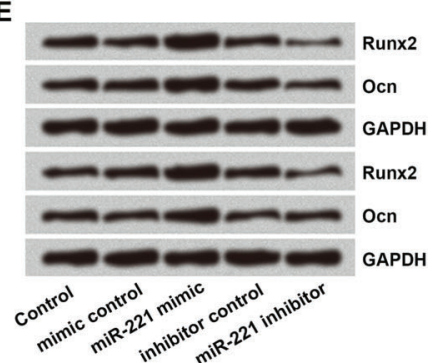

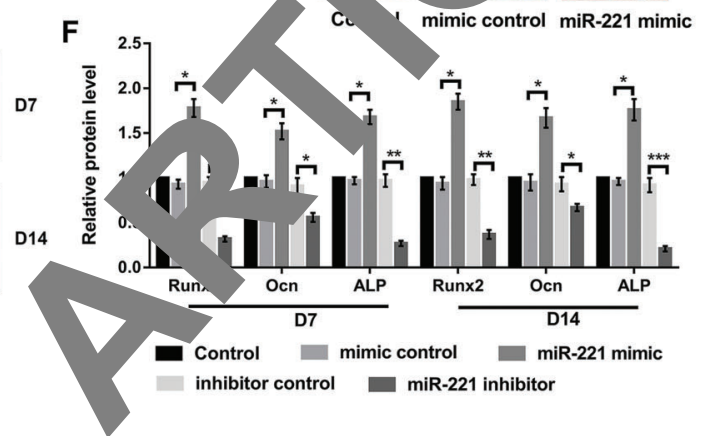

Figure 4. Overexpression of miR-221 promoted osteoblast differ (latio. The mRNA expressions of $(A)$ Runx2 and $(B)$ Ocn were measured using qRT-PCR. C, The mineralized nodule formation MC3T3- cells with miR-221 overexpression was examined using the Alizarin Red S staining assay. D-F, Protein levels of ALP, $P$ nx2, $\quad$ Ocr ere determined by western blot. miR-221: microRNA-221; ALP: alkaline phosphatase; Runx2: runt-related transcript factor 2 , osteocalcin; qRT-PCR: quantitative reverse transcription polymerase chain reaction. Data are reported as means ${ }^{*}{ }^{*} P \quad 0.05,{ }^{*}, 0.01,{ }^{* * *} P<0.001$ (ANOVA).

effect on luciferase activity (Figure 5C). In st o results Overexpression of miR-221 deactivated Wnt/Notch indicated that ZFPM2 was a direct target gene of $\mathrm{h}, 221$, and miR-221 inhibited ZFPM2 expres

Overexpression of miR-221 promo toste last proliferation, migration, and differen. oy targeting ZFPM2

To uncover whether ZFPM va invived in the processes of osteoblast $p$ ratic migration, and differentiation, miR-221 mi $c$ al $\mathrm{pc}-\bar{Z} \quad \mathrm{M} 2$ were transfected into MC3T3-E1 cer o miR-221 and ZFPM2 expression. The sults Figure $6 \mathrm{~A}$ and $\mathrm{B}$ showed that miR-221 overf ssion sis ificantly increased cell viability and migrauon o pared with the mimic control group $(\mathrm{P}<0.05$ sut overex , ession of ZFPM2 reversed these effects de asing cell viability and migration $(P<0.05)$. Western, resi revealed that overexpression of miR22 reas expression of cell cycle-related proteins CN Cyclir A, Cyclin E1, CDK2, Cyclin D1, and CDK4), + ression of ZFPM2 reversed these effects (P 05, Figure 6C and D). Moreover, the expression level of dir entiation-related proteins (ALP, Runx2, and Ocn) were increased by miR-221 mimic, but overexpression of ZFPM2 reversed these effects $(P<0.05$, Figure $6 E$ and $F)$. These findings indicated that overexpression of $m i R-221$ promoted cell viability, migration, and differentiation by regulating the expression of ZFPM2.

\section{and Smad signaling pathways}

Lastly, we measured the effect of miR-221 and ZFPM2 on the Wnt/Notch and Smad signaling pathways using western blot. Overexpression of miR-221 decreased the protein level of Wnt3a, Wnt5a, Notch 1, Notch 2, Notch 3, p-Smad2, Smad4, and Smad7, but overexpression of ZFPM2 reversed these effects ( $P<0.05$ or $P<0.01$, Figure $7 A-D)$. These findings indicated that overexpression of miR-221 deactivated Wnt/Notch and Smad signaling pathways by regulation of ZFPM2.

\section{Discussion}

Bone fracture is a common medical condition, which is damage in the continuity of the bone, and this disease occurs frequently in children and the elderly (17). The activity of osteoblasts is closely related to the quality of recovery of bone fractures. Osteogenesis is a complex and multistep processes, involving the differentiation of mesenchymal stem cells into osteoblast progenitor cells, preosteoblasts, osteoblasts, and osteocytes, as well as crosstalk between multiple cell types for the formation and remodeling of bone (18). The process is regulated by various signaling networks, such as BMP, Wnt ligands, Notch ligands, transforming growth factor (TGF), tumor necrosis factor, and cytokines. A recent study demonstrated 
A Position 295-301 of ZFPM2 3' UTR

hsa-miR-221

B

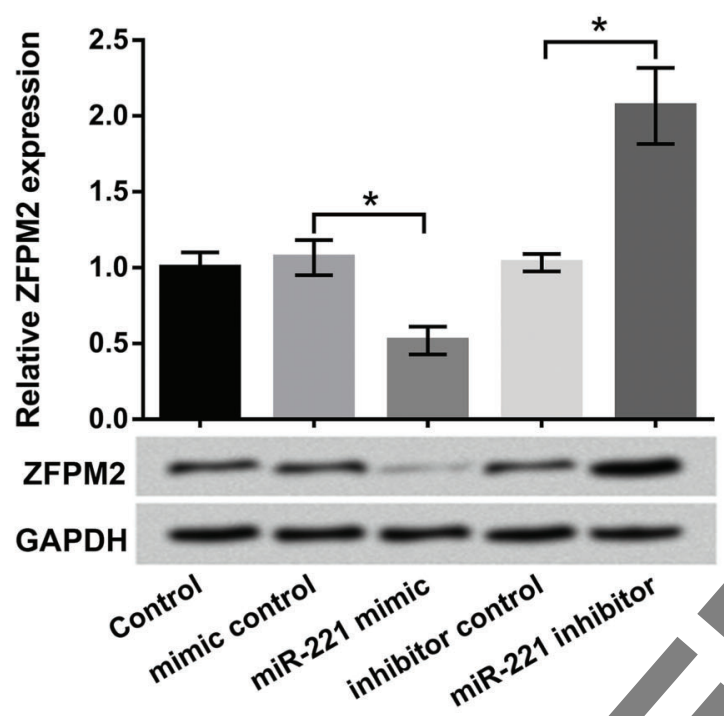

5' ...AUUguaUAGUUAUUGUGUAGCAC.

3' ...UUUgggucgucuguUACAUCGA

C

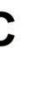

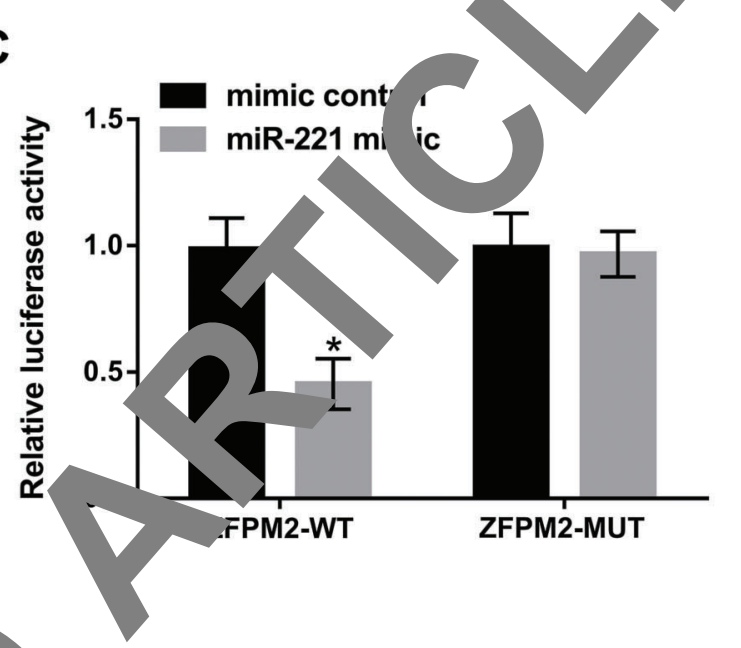

Figure 5. ZFPM2 was a direct target of miR-221. The ndin olte of miR-221 and ZFPM2 was analyzed by TargetScan and microRNA database. $B$, mRNA and protein levels $C$-PM2 we amined by qRT-PCR and western blot. $C$, The relationship between miR-221 and ZFPM2 was detected by dual-lucif ${ }_{\text {SS }}$ orter assay. ZFPM2: zinc finger protein multitype 2; miR-221: microRNA-221; qRT-PCR: quantitative reverse transcription polymerase ain reaction. Data are reported as means \pm SD. ${ }^{*} P<0.05$ (ANOVA).

that miRNAs acted as important reg ators of steogenic signaling pathways (19). In this study, inve gated the effects and mechanism of miR-221 on ulasts proliferation, migration, and differei the results showed that miR-221 was up-regulated putients with LCM and TF, and closely relat ith th orocess of osteoblastic differentiation. Moreo i, w found hat overexpression of miR-221 significantly or proliferation, migration, differentiation, ? ma mineralization in osteoblasts, and supprese of miR- 1 showed contrary results. Further exnerimen showed that ZFPM2 was a direct target of ${ }_{1} \mathrm{R}-221$, an overexpression of miR-221 promoted il vily, migration, and differentiation by downregulatio $Z F$ - 2 . Finally, the results indicated that $m$ ov ression blocked Wnt/Notch and Smad7 gnal a pathwrays by regulating ZFPM2 expression. is one of the important miRNAs, which has be videly reported in various cancers, but it has not been fully in estigated in osteoblastic differentiation (20). Several other miRNAs have been shown to be up-regulated in osteoblastic differentiation. For example, Chen et al. (21) showed that miR-34a was up-regulated in osteoblastic differentiation of human stromal stem cells. Li et al. (22) demonstrated that miR-216a was remarkably up-regulated during osteogenic differentiation in human adipose-derived MSCs. Interestingly, miR-31 was reported to be downregulated during osteoblastic differentiation, however, miR-31 was later identified to be up-regulated during osteoblastic differentiation $(23,24)$. Similar to these studies, we found that miR-221 was up-regulated in LCM and TF patients and also up-regulated during osteoblastic differentiation. These data indicated that miR-221 might be involved in the process of osteoblastic differentiation.

Osteogenic differentiation is divided into four stages: cellular commitment, proliferation, matrix maturation, and mineralization. Our study demonstrated that overexpression of miR-221 promoted osteoblast proliferation. In line with this finding, Xu et al. (25) showed that transfection of MC3T3-E1 osteoblasts with miR-365 ameliorated dexamethasone-induced inhibition of cell viability. Cyclins and CDKs are known to be regulators of cell cycle. An in vitro study has shown that Cyclin E, Cyclin B, Cyclin A, and CDK inhibitors regulate osteoblastic differentiation (26). Our study also found that overexpression of miR-221 increased the expression of cell cycle-related proteins (PCNA, Cyclin A, Cyclin E1, CDK2, Cyclin D1, and CDK4). Thus, these findings indicated that overexpression of miR-221 promoted cell proliferation in osteoblasts. 
A

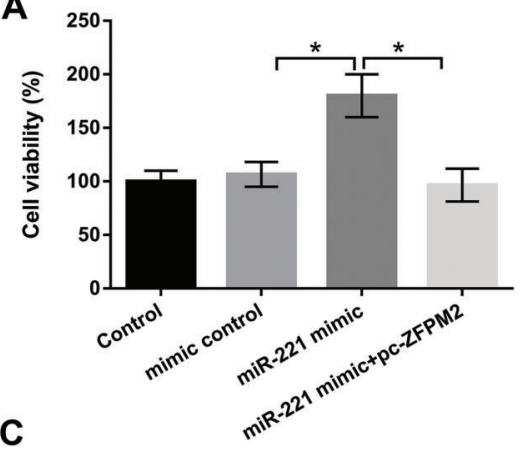

C

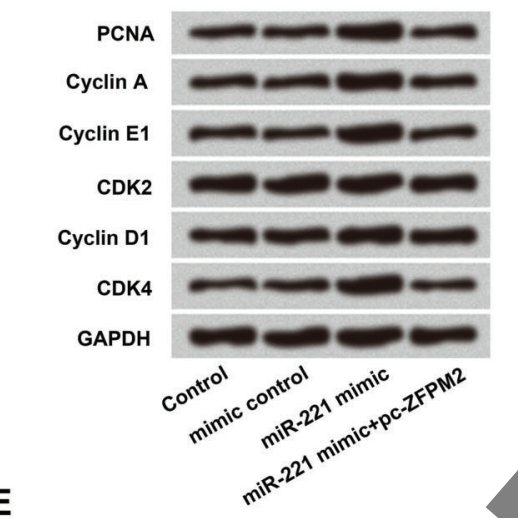

$\mathbf{E}$

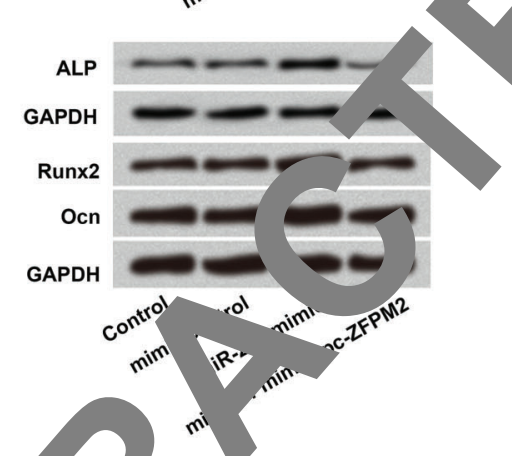

B

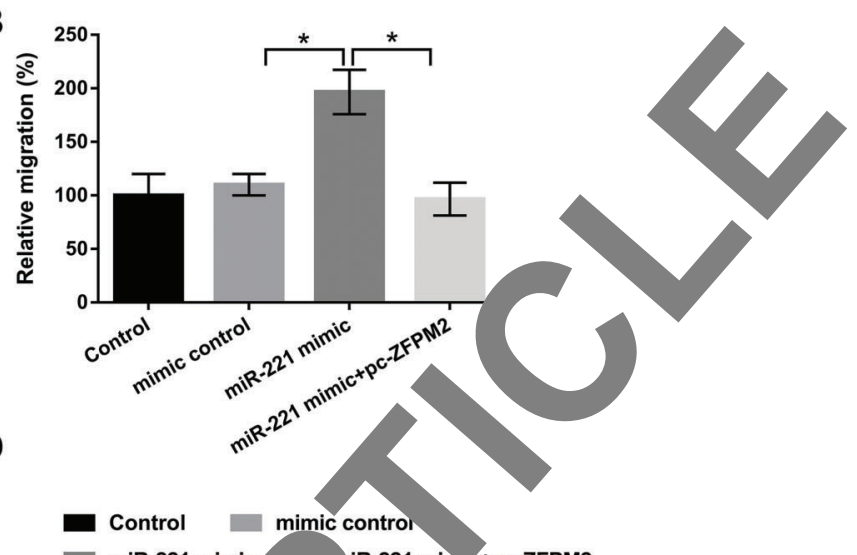

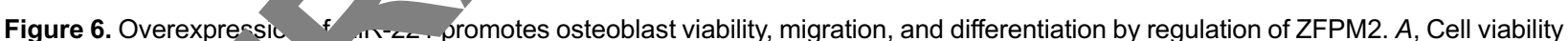
was measured usi the $C$ \& assay. $B$, Cell migration was measured using the Transwell migration assay. $C$ and $D$, Protein levels of cell cycle-relater 'eins wero reasured using western blot. $E$ and $F$, Protein levels of differentiation-related proteins were detected by western blot. W.R-2_ microRNA-221; ZFPM2: zinc finger protein multitype 2; CCK-8: cell counting kit-8; PCNA: proliferating cell nuclear ant' $n$; CDK: $c$, $\eta$-dependent kinase; ALP: alkaline phosphatase; Runx2: runt-related transcription factor 2; Ocn: osteocalcin. Data are port ${ }^{-1}$ as means $\pm S D$. ${ }^{*} P<0.05$ (ANOVA). ns: not significant.

\begin{abstract}
$\mathrm{T}$ is $\mathrm{i}$ gical process, which is characterized by tran tion frum epithelial cells to interstitial phenotypes , orocedures. Mounting evidence has indicated tha $-\mathrm{MT}$ is involved in the formation of many tissues and orgar. during development $(27,28)$. Moreover, several signaling pathways, such as TGF- $\beta$, Wnt, and Notch, have been reported to induce the EMT process. These signaling pathways can activate transcription factors, including Snail, Slug, and ZEB family, which suppress the expression of E-cadherin, resulting in cell invasion and migration (29).
\end{abstract}

Osteoblast migration improves the repair of bone fracture and growth of bone tissue (30). Our study found that overexpression of miR-221 promoted osteoblast migration by decreasing the expression of E-cadherin and increasing the expression of $\mathrm{N}$-cadherin, Vimentin, ZEB1, and Snail.

Understanding the regulatory mechanism of osteoblast differentiation is very important to develop strategies for treating bone disorders, including bone fracture. Runx2, Osterix, and $\beta$-catenin are the vital transcription factors for osteoblast differentiation (6). Runx2 is a main transcription 


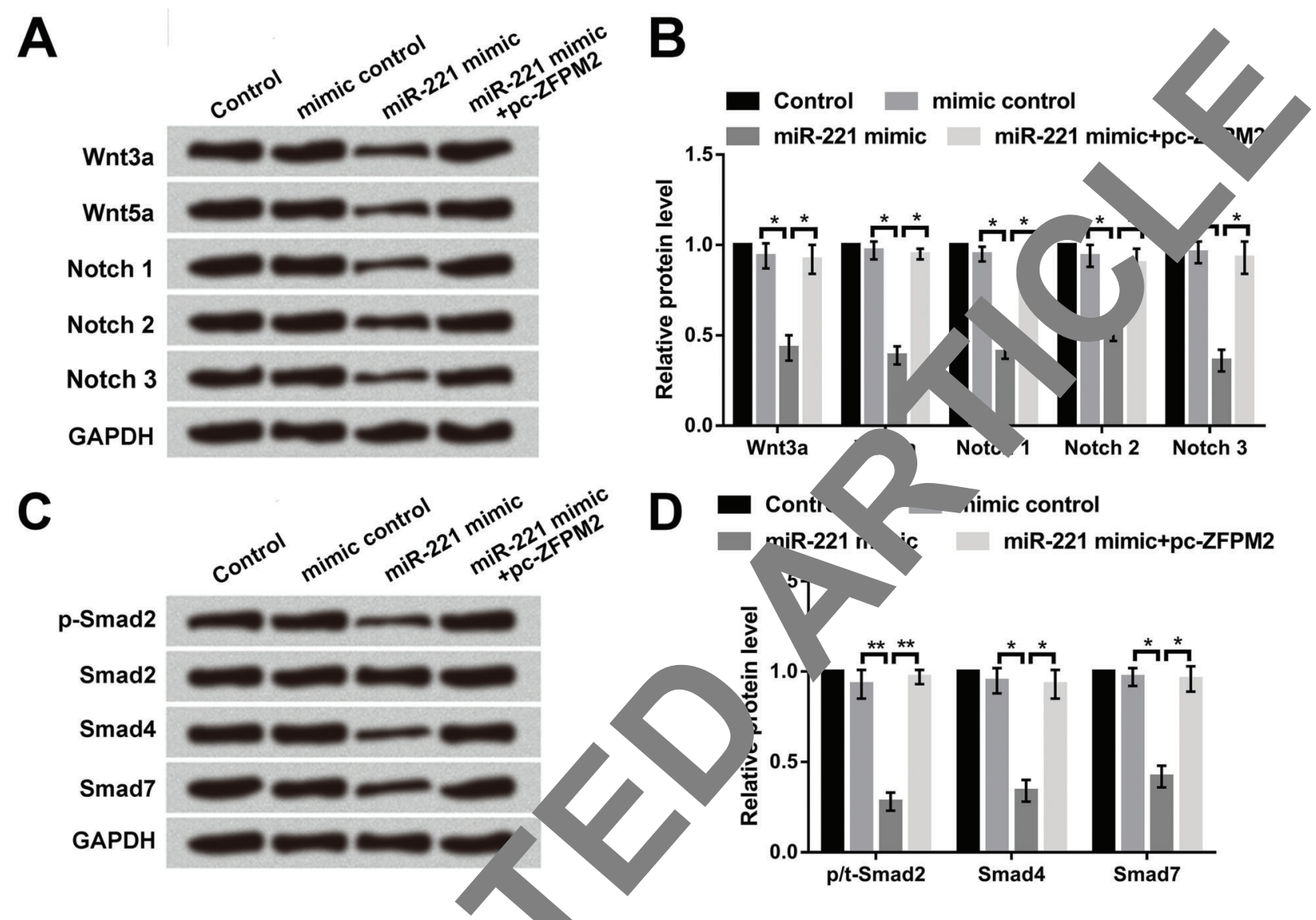

Figure 7. Overexpression of miR-221 dear ed Wnt/Nu in and Smad signaling pathways. MC3T3-E1 cells were transfected with mimic control, miR-221 mimic, or miR-22 nimic + pc-ZFPM2. The protein levels of $(A$ and $B)$ Wnt3a, Wnt5a, Notch 1 , Notch 2 , and Notch 3, and $(C$ and $D)$ Smad2, Smad4, a Smad 1 ere determined using western blot. miR-221: microRNA-221; zFPM2: Zinc finger protein multitype 2. Data are reported as $\mathrm{h}$. $n \pm^{c}$. ${ }^{*} \mathrm{P}<0.05,{ }^{* *} \mathrm{P}<0.01$ (ANOVA).

factor required for the differe, in osteoblasts from mesenchymal precursors and ut quunt bone matrix mineralization (31). Mor rec research has proven that Runx2 can direr st ulate le osteoblast marker gene expression, su a ocn is a late bone marker, which ? Jears osteogenic differentiation and mineralization Several dies have shown the involvement of miRN As in "ooblast differentiation. For example, miR-26a id miR-12 are shown to inhibit osteoblast differer cion nereas miR-33-5p and miR-194 are reported to prom stec ast differentiation $(34,35)$. An interestin dy 2 -nang et al. (36) reported that miR-221 suld hibit outeogenic differentiation by targeting Runx2 Similarly, Yeh et al. (37) found that miR-221 at ated the osteogenic differentiation in human annulus fic osus cells. However, the opposite results in the present study revealed that miR-221 promoted osteoblast differentiation by increasing the expression of ALP, Runx2, and Ocn in MC3T3-E1 cells. The different results might be related to the different cell lines used. Further studies are still needed to confirm the hypothesis.
ZFPM2 is a zinc finger protein encoded by the ZFPM2 gene, which is an important regulator of hematopoiesis and cardiogenesis in mammals (38). A recent study revealed that miR-429 could induce MC3T3-E1 osteoblastic cells differentiation by regulation of ZFPM2 expression (39). However, whether miR-221 affects cell proliferation, migration, and differentiation through regulating ZFPM2 expression in MC3T3-E1 cells is still unclear. In our study, we found that ZFPM2 was a direct target of miR-221. Moreover, miR-221 decreased the expression of ZFPM2 in osteoblasts. Further experiments revealed that overexpression of ZFPM2 reversed the promoting effects of miR-221 on MC3T3-E1 cells proliferation, migration, and differentiation, indicating that the effects of miR-221 on osteoblastic cells are mediated via regulating ZFPM2.

It has been reported that Wnt, Notch, and Smad signaling pathways play important roles in osteoblast differentiation (40). Therefore, we explored the effect of miR-221 and ZFPM2 on Wnt/Notch and Smad signaling pathway proteins (Wnt3a, Wnt5a, Notch 1 to 3, Smad2, Smad4, and Smad7), and found that overexpression of miR-221 
decreased the protein levels of these proteins, but ZFPM2 overexpression reversed these effects, indicating that miR-221 blocked Wnt/Notch and Smad signaling pathways by regulation of ZFPM2.

In conclusion, these results revealed that miR-221 was up-regulated during osteoblastic differentiation, and overexpression of miR-221 promoted cell viability, migration, and differentiation by regulating ZFPM2 expression and deactivating the Wnt/Notch and Smad signaling pathways.

\section{References}

1. Jin H, Wang B, Li J, Xie W, Mao Q, Li S, et al. Anti-DKK1 antibody promotes bone fracture healing through activation of beta-catenin signaling. Bone 2015; 71: 63-75, doi: 10.1016/j.bone.2014.07.039.

2. Kanis JA, Oden A, McCloskey EV, Johansson H, Wahl DACooper C. A systematic review of hip fracture incidence and probability of fracture worldwide. Osteoporos Int 2012; 23: 2239-2256, doi: 10.1007/s00198-012-1964-3.

3. Zura R, Xiong Z, Einhorn T, Watson JT, Ostrum RF, Prayson $\mathrm{MJ}$, et al. Epidemiology of fracture nonunion in 18 human bones. JAMA Surg 2016; 151: e162775, doi: 10.1001/ jamasurg.2016.2775.

4. Ghiasi MS, Chen J, Vaziri A, Rodriguez EK, Nazarian A Bone fracture healing in mechanobiological modeling: A review of principles and methods. Bone Rep 20 87-100, doi: 10.1016/j.bonr.2017.03.002.

5. Histing T, Stenger D, Kuntz S, Scheuer C, Tami A et al. Increased osteoblast and osteoclast acti in senescence-accelerated, osteoporotic SAM' 'ce durin fracture healing. J Surg Res 2012; 175. 271 7 , doi: 10.1016/j.jss.2011.03.052.

6. Komori T. Regulation of osteoblast iferentiation by transcription factors. J Cell Biochem 06; 99: 233-1239, doi: 10.1002/jcb.20958.

7. Guntur AR, Rosen CJ. New mights in weoblasts and their role in bone formation: role of PI3Kinase. J Endocrinol 2011; 211: 123-1 d 10.1530/JOE-11-0175.

8. Karsenty G, Kronenb MM, S embre C. Genetic control of bone formation. inu ev Ce. Jev Biol 2009; 25: 629648, doi: 10.1146 nur 042308.113308 .

9. Piccoli MT, Gr a S, num T. Noncoding RNAs as regulators of carr nyocyte liferation and death. $\mathrm{J} \mathrm{Mol} \mathrm{Cell}$ Cardiol 2 o; 59-67, aoi: 10.1016/j.yjmcc.2015.02.002.

10. Bartel Micror 's: genomics, biogenesis, mechanism, and iction. Cell 20u4; 116: 281-297, doi: 10.1016/S00928 (04) $045-5$

11. Dors Yand, Guo H, Kang F. MicroRNAs regulate osteonesis chondrogenesis. Biochem Biophys Res Comn 2012, 418: 587-591, doi: 10.1016/j.bbrc.2012.01.075. latwong S. MicroRNAs in bone development and heir diagnostic and therapeutic potentials in osteoporosis. anect Tissue Res 2017; 58: 90-102, doi: 10.3109/ 03008207.2016.1139580.

13. Waki T, Lee SY, Niikura T, Iwakura T, Dogaki Y, Okumachi E, et al. Profiling microRNA expression during fracture healing. BMC Musculoskelet Disord 2016; 17: 83, doi: 10.1186/ s12891-016-0931-0.
Our novel findings indicate a potential role of miR-221 in osteoblast proliferation, migration, and differentia $n$.

\section{Acknowledgments}

This study was funded in full by the Key Laborat of Tumor Molecular Biology of Ningbo C. (2015f 2011) and the Key Subjects of Ningb o.2 al (No. 2016-55).
14. Nakasa T, Yoshizuk ndry Ush an M, Elbadry Mahmoud E, Ochi M. MicroRNAs d Bone Regeneration. Curr Genomics $20 \quad$ 6: $441-{ }_{4}$, 2, doi: $10.2174 / 138920291$ 666615081,36

15. Yang F, Wo W Xi W, Yuan L, Chen X, et al. MiR$221 / 222$ prom human glioma cell invasion and angiogenesis araeting IP2. Tumour Biol 2015; 36: 3763-3773, doi: Tu $0077-014-3017-3$.

16. Xu Q, $P$, hen $X$, Liang Z, Jiang Z, Nan L, et al. miR-221/ 222 inc es pancreatic cancer progression through the regulatio f matrix metalloproteinases. Oncotarget 2015; 6: 1153-14164, doi: 10.18632/oncotarget.3686.

17. rescu PH, Izvernariu DA, lancu C, Dinu GO, Berceanuluva MM, Crisan D, et al. Pathological fracture of the mur in a patient with Paget's disease of bone: a case report. Rom J Morphol Embryol 2016; 57: 595-600.

3. Lian JB, Stein GS, van Wijnen AJ, Stein JL, Hassan MQ, Gaur T, et al. MicroRNA control of bone formation and homeostasis. Nat Rev Endocrinol 2012; 8: 212-227, doi: 10.1038/nrendo.2011.234.

19. Yuan Z, Li Q, Luo S, Liu Z, Luo D, Zhang B, et al. PPARgamma and Wnt signaling in adipogenic and osteogenic differentiation of mesenchymal stem cells. Curr Stem Cell Res Ther 2016; 11: 216-225, doi: 10.2174/1574888X 10666150519093429.

20. Li T, Li M, Hu S, Cheng X, Gao Y, Jiang S, et al. MiR-221 mediates the epithelial-mesenchymal transition of hepatocellular carcinoma by targeting AdipoR1. Int J Biol Macromol 2017; 103: 1054-1061, doi: 10.1016/j.ijbiomac.2017.05.108.

21. Chen L, Holmstrom K, Qiu W, Ditzel N, Shi K, Hokland L, et al. MicroRNA-34a inhibits osteoblast differentiation and in vivo bone formation of human stromal stem cells. Stem Cells 2014; 32: 902-912, doi: 10.1002/stem.1615.

22. Li H, Li T, Fan J, Li T, Fan L, Wang S, et al. miR-216a rescues dexamethasone suppression of osteogenesis, promotes osteoblast differentiation and enhances bone formation, by regulating c-Cbl-mediated PI3K/AKT pathway. Cell Death Differ 2015; 22: 1935-1945, doi: 10.1038/cdd.2015.99.

23. Gao J, Yang T, Han J, Yan K, Qiu X, Zhou Y, et al. MicroRNA expression during osteogenic differentiation of human multipotent mesenchymal stromal cells from bone marrow. J Cell Biochem 2011; 112: 1844-1856, doi: 10.1002/jcb.23106.

24. Baglio SR, Devescovi V, Granchi D, Baldini N. MicroRNA expression profiling of human bone marrow mesenchymal stem cells during osteogenic differentiation reveals Osterix regulation by miR-31. Gene 2013; 527: 321-331, doi: 10.1016/ j.gene.2013.06.021. 
25. Xu D, Gao Y, Hu N, Wu L, Chen Q. miR-365 ameliorates dexamethasone-induced suppression of osteogenesis in MC3T3-E1 Cells by targeting HDAC4. Int J Mol Sci 2017; 18: pii: E977, doi: 10.3390/ijms18050977.

26. Drissi H, Hushka D, Aslam F, Nguyen Q, Buffone E, Koff A, et al. The cell cycle regulator p27kip1 contributes to growth and differentiation of osteoblasts. Cancer Res 1999; 59: 3705-3711.

27. Xu MH, Gao X, Luo D, Zhou XD, Xiong W, Liu GX. EMT and acquisition of stem cell-like properties are involved in spontaneous formation of tumorigenic hybrids between lung cancer and bone marrow-derived mesenchymal stem cells. Plos One 2014; 9: e87893, doi: 10.1371/journal.pone.0087893.

28. Shimizu $M$, Kondo S, Urushihara $M$, Takamatsu $M$, Kanemoto K, Nagata M, et al. Role of integrin-linked kinase in epithelial-mesenchymal transition in crescent formation of experimental glomerulonephritis. Nephrol Dial Transplant 2006; 21: 2380-2390, doi: 10.1093/ndt/gfl243.

29. Son H, Moon A. Epithelial-mesenchymal Transition and Cell Invasion. Toxicol Res 2010; 26: 245-252, doi: 10.5487/ TR.2010.26.4.245.

30. Riehl BD, Lee JS, Ha L, Kwon IK, Lim JY. Flowtaxis of osteoblast migration under fluid shear and the effect of RhoA kinase silencing. PLoS One 2017; 12: e0171857, doi: 10.1371/journal.pone.0171857.

31. Tamargo J, Caballero R, Delpón E. The renin-angiotensin system and bone. Clin Rev Bone Mineral Metab 2015; 13 . 125-148, doi: 10.1007/s12018-015-9189-6.

32. Fei L, Wang C, Xue Y, Lin K, Chang J, Sun J. Oste senic differentiation of osteoblasts induced by calcium sil a calcium silicate/beta-tricalcium phosphate cor osiu ceramics. J Biomed Mater Res B Appl Biome 1237-1244, doi: 10.1002/jbm.b.32688.
33. Granéli $\mathrm{C}$, Thorfve A, Ruetschi U, Brisby $\mathrm{H}$, Thomsen $\mathrm{P}$, Lindahl $A$, et al. Novel markers of osteogenic ar adipogenic differentiation of human bone marrow $s^{\prime}$ inal cells identified using a quantitative proteomics ap act stem Cell Res 2014; 12: 153-165, doi: 10.1016/j.scr.2u 9.00

34. Mizuno Y, Yagi K, Tokuzawa Y, Kanesaki-Yatsuka Y, $T$, Katagiri T, et al. miR-125b inhibits oste stic differentration by down-regulation of cell proliferation. cher siophys Res Commun 2008; 368: 267 , doi: 16/j.bbrc. 2008.01.073.

35. Wang $\mathrm{H}$, Sun Z, Wang $\mathrm{Y}, \mathrm{Hu} Z$, , ou $\mathrm{H}, \mathrm{Z}$, $\mathrm{gg} \mathrm{L}$, et al. miR33-5p, a novel mechano-se. 'ive promotes osteoblast differentiation by get. Hmgaz. Sci Rep 2016; 6: 23170, doi: 10.1038

36. Zhang Y, Gao Y, $C, L$, Lou Y, Xu N, et al. MicroRNA221 is involved in the rey tion of osteoporosis through regulates $\mathrm{RU}^{\prime} \mathrm{C}$ otein expression and osteoblast differentiation. f $J$ Tr al Res 2017; 9: 126-135.

37. Yeh $\mathrm{CH}$, Jir the osteogenic rentiation of human annulus fibrosus cells. Spir 16: o J-904, doi: 10.1016/j.spinee.2016.03.026.

38. Li TP, uke in G, Sheu T, Maruyama T, Jonason JH, et al. B $\angle$ induces ATF4 phosphorylation in chondrocytes through $\mathrm{OX}-2 / \mathrm{PGE} 2$ dependent signaling pathway. Osteoarthritis Cartage 2014; 22: 481-489, doi: 10.1016/j.joca.2013. 020.

39. A ing J, Peng J, Cao G, Lu S, Liu L, Li Z, et al. Hypoxiauced MicroRNA-429 Promotes Differentiation of MC3T31 Osteoblastic Cells by Mediating ZFPM2 Expression. Cell Physi Biochem 2016; 39: 1177-1186, doi: 10.1159/000447824.

0. Lin GL, Hankenson KD. Integration of BMP, Wnt, and notch signaling pathways in osteoblast differentiation. J Cell Biochem 2011; 112: 3491-3501, doi: 10.1002/jcb.23287. 